\title{
AN INTELLIGENT LOCATION AND STATE REORGANIZATION OF TRAFFIC SIGNAL
}

\author{
Saeed BEHZADI \\ Department of Surveying Engineering, Faculty of Civil Engineering, \\ Shahid Rajaee Teacher Training University, Lavizan, Tehran, Iran
}

Received 14 July 2019; accepted 15 September 2020

\begin{abstract}
In all geo-database related to traffic, beside storing roads data, the information associated to traffic signals such as location, types of traffic signals, side street name, and so on are also stored in that database. In reality, the reason of defining traffic signals for road is the situations and conditions which the roads have. So the existence of traffic signals in the network is related to the parameters of the road. In this paper, instead of storing traffic signal data in the database, a novel method is introduced which implemented on the road network. As a result, the spatial and non-spatial information of traffic signals in the network are extracted based on the location and attribute of the road network. The proposed method is implemented on the network; the result of the intelligent method is compared with the traffic signals information which stored in the database. By comparing the locations and states of proposed traffic signals and the real ones, the overall accuracy for recognizing locations of traffic signal is obtained $94 \%$ and the overall accuracy for recognizing states of traffic signal is obtained $89 \%$.
\end{abstract}

Keyword: Geospatial Information Systems (GIS), network, road, traffic signal.

\section{Introduction}

One of the most important problems which municipality is faced is traffic. Traffic information consists of spatial and non-spatial ones. Temporality is also another quality of the traffic. Recently, one of the most important challenges for Geospatial Information Systems (GIS) is the storing and visualizing spatial and non-spatial information of traffic (Jabbari \& Behzadi, 2019). Traffic data are limited to traffic system engineers. The full potential and benefit of historical traffic databases could be expanded by an integration of traffic system data in GIS. A traffic GIS might act as a reference database and as s support for spatiotemporal analysis (Behzadi \& Alesheikh, 2014; $\mathrm{Li}$ et al., 2019). Integrating traffic data with GIS is widely considered by some researchers. The IMPACT (integrated model for population ageing consequences on transportation) model, introduced by Maoh et al. (2009), simulated the future of travel demand in urban areas. In the proposed method, travel demand models had been developed based on demographic changes and the ageing of population. The GIS, used in this paper, is considered for assessing the ramifications of demographic changes, including population ageing, on the performance and usage of urban transportation systems. In this paper, the property of storing and visualizing of GIS is used. Ying (2007) proposed an integrated land use/transportation model. In the model, sensitivity-analysis-based techniques are used for the optimal design of land use plans and transportation policies. In this paper, GIS and transportation networks are considered as two sub-models which mutually contract with each other. Like the previous research, GIS is used as simple database. Clay and Johnston also used GIS for storing integrated land use and transportation forecasting model. The model monitored the impacts of uncertainty on outputs, and determined which sources of uncertainty have the largest impact on these outputs (Clay \& Johnston, 2006). In the paper proposed by Maria and Gleriani (2005), a cellular automaton (CA) simulation on urban land use change was introduced. In this paper, a supervised back propagation neural network had been employed in the parameterization of the simulation model. The proposed model had been tested in a mediumsized town. In the other researches such as Shaw and Xin (2003) and Wang (2005), the integrated of GIS and traffic are also used.

Recently, researchers have attempted to develop effective intelligent systems to assist managers in making

*Corresponding author. E-mail: behzadi.saeed@gmail.com 
decisions about how to solve various problems (Fraile et al., 2016; Ocalir-Akunal, 2016). Based on this idea, Intelligence Transportation System (ITS) is expressed in traffic field to manage the traffic problem automatically. In the paper proposed by Srinivasan (Srinivasan et al., 2006), the hybrid NN-based multi-agent system approach was used to develop distributed unsupervised traffic responsive signal control models. In this paper, each agent was considered as a local traffic signal controller for one intersection in the traffic network. Each agent had eight different types of signal plans that it could use to control the traffic signals at its intersection. The result of the experiences showed that the mean delay of each vehicle was reduced by $78 \%$ by using the proposed type of multi-agent system. Moreover, the mean stoppage time was reduced by $85 \%$ compared to the existing traffic signal control algorithm. In the paper presented by Ozkurt et al. (2009) the classification of vehicles and calculation of traffic density is assessed by using neural networks. In this paper, traffic density is automatically estimated. In addition, vehicles are classified through video processing and artificial systems. 'Awad (2004) suggested an alternative and convenient procedure for estimating capacity on weaving segments. In this paper, two capacity prediction models were developed using regression and neural networks (NNT). The result of the experience proved that linear regression (LR) technique showed satisfactory results; however, neural network technique outscored linear regression in the prediction performance, and generalization ability. In the papers proposed by Mao et al. (2008) (predicting traffic volum), Annunziato et al. (2003) (Predicing Traffic Flow) the traffic parameterd are controlled by using computational inteligence. Most of these researches are done to use intelligent transportation system to control traffic in the environment. It might rarely be found research for using ITS for storing traffic data in the database.

In addition, the Automatic Target Recognition (ATR) is a considerable interest in many industrial applications. Some constraints, such as real time response, high detection accuracy, adaptive capabilities to noisy environments, increase the complexity of the target detection task (Al Mufti et al., 2018). These methods can be used in many applications and investigated based on the conditions of the problem.

In this paper, to store traffic data in the geo database, an intelligent method is introduced, which recognizes the traffic signal data from road data. Road information is the foundation of the traffic geo-database. The reason of storing the traffic signal on the road is related to the density and the other parameters of the road. However, in all traffic geo-databases, traffic signal is stored as separate information in the data base while in reality the existence of the traffic signal in the network is interrelated to the road of that network. In this paper, an intelligent method is proposed based on the essence of existent of the traffic signal in the network. The following sections explain the theory of the method, and then the proposed method is implemented on the experimental network. Finally, the result is investigated and compared with the real traffic signal stored in the database.

\section{Information of the traffic signal}

In all geo databases, traffic signal information is stored in the database as a separate data which contains spatial and non-spatial information. As the existence of the traffic signal in the network depends on the roads and its situation, the existence of the traffic signal in the database can depend on the road information stored in the database. All roads data in the database have spatial and non-spatial information from which the spatial and non-spatial information of the traffic signal can be extracted. The method of extracting the information of the traffic signal can be divided to two parts will be discussed in this part.

\section{Spatial information of traffic signal}

The spatial information of traffic signal is the number of traffic signal along with their X, Y coordinates. The road information is used in the network to find the number of traffic signals. Each parcel of road in the database has two coordinates related to the both ends of the parcel. All these coordinates of roads are searched. If three or more of these coordinates are the same, it shows that an intersection might be located at that X, Y coordinates. So these similar coordinates along with the number of the repetitions are extracted from the road network.

In common databases, the traffic signals stored in the database only have coordinates and there is no sign of relation between the traffic signal and road in these types of databases. To find which traffic signal relates to which road, some operations such as "buffer" are used. The roads related to the traffic signal are specified by defining buffer operation around traffic signal and intersecting the buffer area and roads. In the proposed method, a unique number is assigned for the same road at each intersection. This number is stored as a new field in the road data. In this way, all roads having the same traffic signal are specified by searching the specific number in the road data.

Specifying the side of the traffic signal for road is the other parameter which must be considered for traffic signal. Based on the type of traffic signal (four-way or threeway), each traffic signal has four or three faces related to the road connected to the traffic signal. Specifying the sides of traffic signal for roads helps the decision maker to assign the same state of the signal for the roads in the same direction. In many situations, the roads located in front of each other at the specific intersection have the same state of traffic signal. A new type of encoding is introduced to specify the road with approximately the same direction. All roads with the same traffic signal have the same integer identification. For these roads, a decimal number is added which shows the side of the traffic signal. By following this rule, if the roads are in the same direction, the decimal number must be $0.1,0.3,0.5$, etc. or $0.2,0.4,0.6$, etc. 


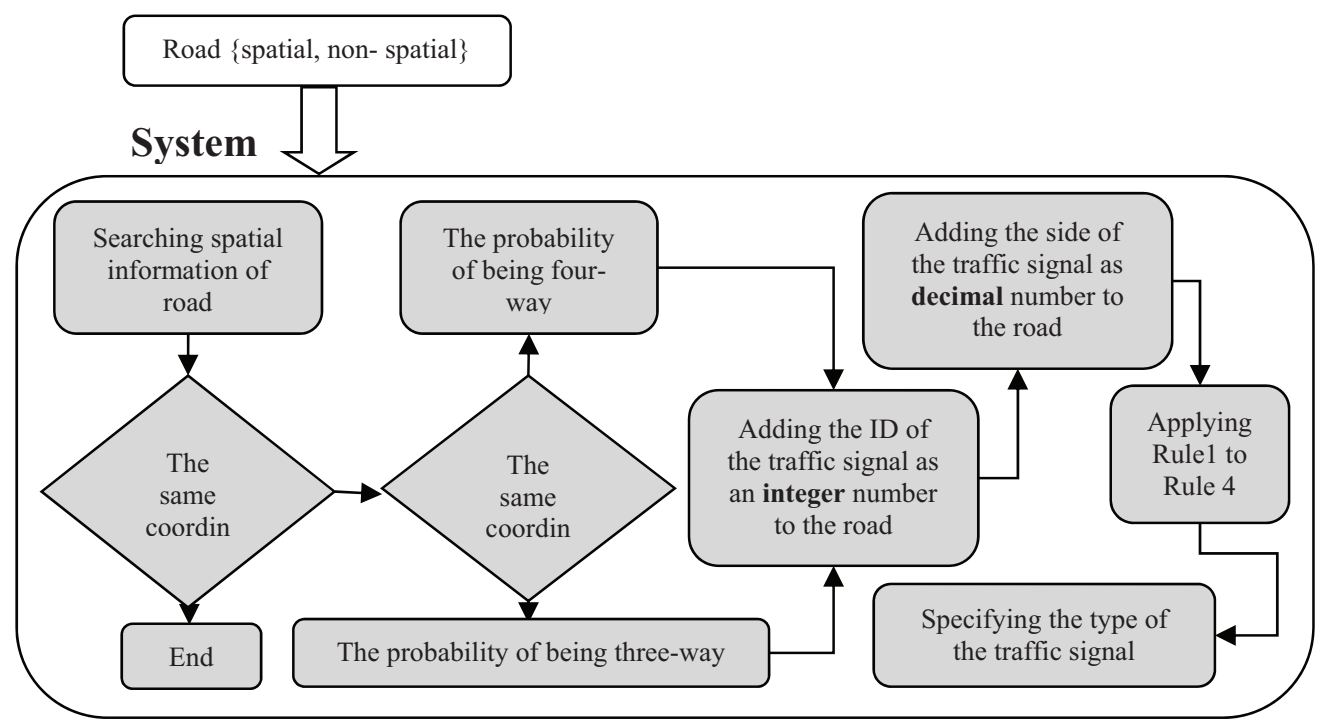

Figure 1. The structure of proposed method

It rarely happens that a traffic signal faces with more than four roads, but by using this identification, nine roads can be supported. By using such encoding, a real number is assigned for each road, containing integer and decimal parts. The integer part shows the ID of the traffic signal and the decimal part shows the side of the traffic signal. So the spatial information of the traffic signal is specified while there is no need for adding a separate data for traffic signal. The flowchart of the proposed algorithm is shown in Figure 1.

\section{Non-spatial information of traffic signal}

The most important non spatial information for the traffic signal is the type and states of traffic signal for connected roads. Annual average daily traffic (AADT) is the most common information stored for road data (Fu et al., 2017). Most of the decisions made in traffic are based on all AADT's of roads. So such information is used for specifying the type and states of the traffic signal. In the traffic signal database, AADT is assigned for each road, which shows the annual average daily traffic of that road. This information is extracted from all roads and used as input data for the proposed method. To find the type and state of the traffic signal, the following process must be done:

1. For each traffic signal, the connected roads are found (the real part of the number associated to road in the previous section shows the identification of the traffic signal).

2. All roads selected for each traffic signal is divided into two parts based on the direction of the roads. The decimal parts of the roads show the direction of the roads (the roads with the decimal number such as $0.1,0.3,0.5$, etc. or $0.2,0.4,0.6$, etc. are in the same direction).

3. The average of AADT for all roads related to same direction is calculated; as a result, two numbers are obtained for each intersection.
4. The two numbers obtained from the stage 3 named $\mathrm{AADT}_{1}$ and $\mathrm{AADT}_{2}$. Based on $\mathrm{AADT}_{1}$ and $\mathrm{AADT}_{2}$ of each intersection $m$ (the mean of two AADTs at the intersection) and $\delta$ (the standard deviation of two AADTs at the intersection) are calculated. Finally, type of each traffic signal is specified by using the following rules:

Rule 0: if $\left(m<T^{\prime}\right)$, there is no need for defining traffic signal in the intersection.

Rule 1: if $\left(m<T_{1}\right.$ and $\left.\delta<T_{2}\right)$, the state of the traffic signal must be "Amber-Red" blinker.

Rule 1-1: if $\left(\mathrm{AADT}_{1}>\mathrm{AADT}_{2}\right)$, the face of traffic signal to roads related to $\mathrm{AADT}_{1}$ and $\mathrm{AADT}_{2}$ must be "Amber" and "red" respectively.

Rule 1-2: if $\left.\left(\mathrm{AADT}_{1}<\mathrm{AADT}\right)_{2}\right)$, the face of traffic signal to roads related to $\mathrm{AADT}_{2}$ and $\mathrm{AADT}_{1}$ must be "Amber" and "red" respectively.

Rule 2: if $\left(m<T_{1}\right.$ and $\left.\delta>T_{2}\right)$, the state of the traffic signal must be "Green-Amber-Red"

Rule 2-1: the time associated for being green or red depends on $\mathrm{AADT}_{1}$ and $\mathrm{AADT}_{2}$; it also depends on the ratio between $\mathrm{AADT}_{1}$ and $\mathrm{AADT}_{2}$.

Rule 3: if $\left(m>T_{1}\right.$ and $\left.\delta<T_{2}\right)$, the state of the traffic signal must be "Green-Amber-Red". As $\delta$ value is small, the green time associated to roads related to $\mathrm{AADT}_{1}$ and $\mathrm{AADT}_{2}$ must be the same.

Rule 4: if $\left(m>T_{1}\right.$ and $\left.\delta>T_{2}\right)$, the state of the traffic signal must be "Green-Amber-Red".

Rule 4-1: the time associated for being green or red depends on $\mathrm{AADT}_{1}$ and $\mathrm{AADT}_{2}$; it also depends on the ratio between $\mathrm{AADT}_{1}$ and $\mathrm{AADT}_{2}$.

In the proposed rules, there are three unknown variables $T^{\prime}, T_{1}$ and $T_{2} . T^{\prime}$ is a threshold for defining whether there must be traffic signal in the intersection or not. If the average of AADTs in the intersection is less than this threshold, the density in the intersection is not enough for defining a traffic signal. $T_{1}$ is a threshold for finding 
whether traffic signal is blinker or steady. $T_{2}$ is a threshold for standard deviation. If the ratios of departure of vehicles for green, Amber blinker, Amber, red blinker and red are considered $A_{1}, A_{2}, A_{3}, A_{4}$, and $A_{5}$ respectively. $T_{2}$ parameter is obtained by using following equation:

$\bar{A}=\frac{A_{2}+A_{4}}{2} \Rightarrow 2 \bar{A}=A_{2}+A_{4} \Rightarrow A_{4}-\bar{A}=\bar{A}-A_{2} ;$

$T_{2}=1 / 2(M / \bar{A})\left(\left|A_{2}-\bar{A}\right|+\left|A_{4}-\bar{A}\right|\right)$

$\stackrel{E q \cdot 1}{\longrightarrow} T_{2}=1 / 2(M / \bar{A})\left(\left|A_{2}-\bar{A}\right|+\left|\bar{A}-A_{2}\right|\right)=(M / \bar{A})\left|A_{2}-\bar{A}\right|$.

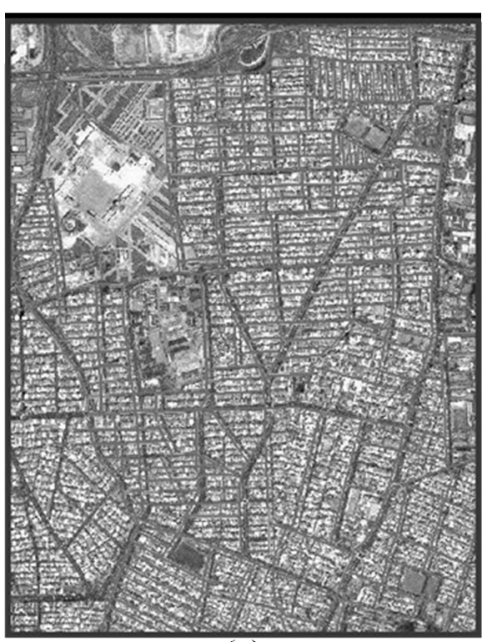

(a)

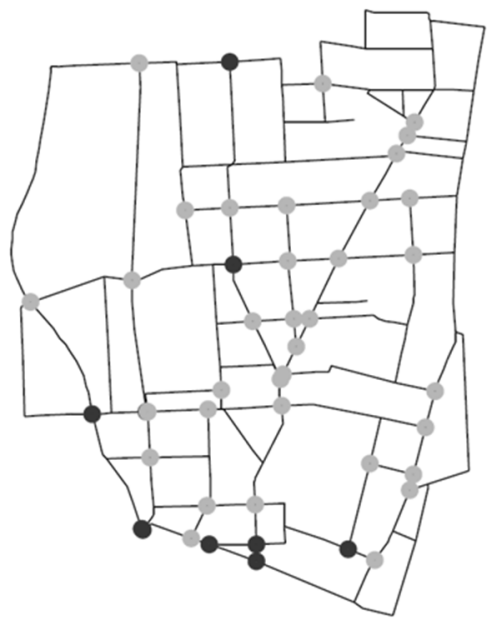

(c)

\section{Implementation}

In the aim to confirm the proposed structures, the method was implemented on the study area. The study area consist of near 180 roads (Figure 2a). Real traffic signals also exist in the data base for evaluating the proposed method (Figure 2b). The method was implemented on the road network to obtain spatial and non-spatial information of traffic signals. The information obtained by proposed method is shown in Figure 2c. The difference between traffic signals obtained by proposing method and the ones stored in the database is shown in Figure $2 \mathrm{~d}$. The point illustrated by $(\square)$ in Figure 2 d shows the points which exist in the real database or in the proposed method but

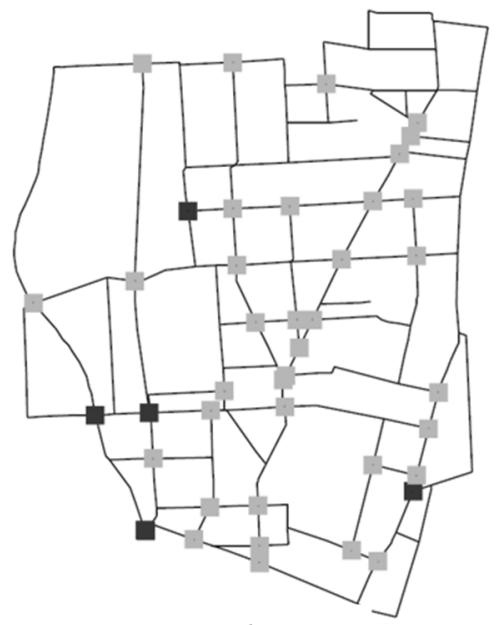

(b)

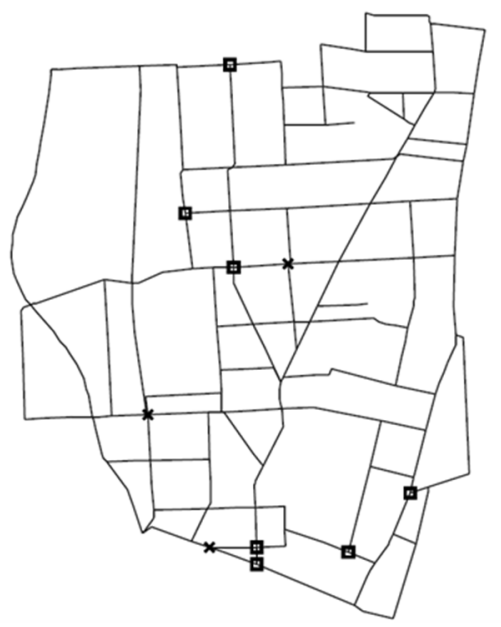

(d)

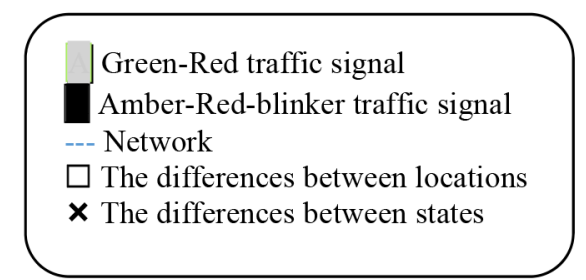

Figure 2. a) The study area, b) the real location and state of the traffic signals, c) the location and state of the proposed traffic signals, d) the comparison between the proposed method and real traffic signal 
they are not in another one. The points illustrated by $(x)$ shows that the proposed method specified the wrong state for traffic signals. The quantitative comparison (error matrix) between proposed method and real data stored in the database is shown in Table 1 and 2.

In Table 1, the locations of traffic signals obtained by proposed model are compared with the real locations. As seen in this table, 42 locations of traffic signals are specified correctly, however, the model select 3 points as the locations of traffic signals while in reality there are no traffic signals in those points. The proposed model also specifies correctly 2 locations as non-traffic signals. In Table 2 , the states of traffic signals obtained by proposed model are compared with the real state. In this table, the model specifies 42 states (31 "Green-Red" states and 11 "Amber Blinker" states) correctly. It also specifies 2 states of traffic signals "Green-Red" while the real states are "Amber Blinker", and 3 states of traffic signal are "Amber Blinker" while the real states are "Green-Red". The overall accuracy for recognizing locations of traffic signals is obtained $94 \%$ and the overall accuracy for recognizing states of traffic signal is obtained $89 \%$.

There are some differences between the result obtained by the proposed method and data stored in the data base. There are two reasons for the differences: first, the position and state of the traffic signals in reality are experimental. There are defined by traffic engineers, and there are no rational rules for defining the position and the state of the traffic signal. As a result, most of them are defined human-based and manually. If the states and locations of traffic signals are defined rationally in reality, the similarity between the proposed method and reality will increase.

Second, the definition of three threshold parameters $T^{\prime}, T_{1}$ and $T_{2}$ depends on user. Although $T_{2}$ is obtained mathematically, the value assigned for $T^{\prime}$ and $T_{1}$ are

Table 1. The comparison of traffic signal location between proposed method and the real once

\begin{tabular}{|c|c|c|c|c|}
\hline & \multicolumn{3}{|c|}{ Real data (Location) } & \\
\hline \multirow{4}{*}{$\begin{array}{l}\text { The } \\
\text { pro- } \\
\text { posed } \\
\text { me- } \\
\text { thod } \\
\text { (Loca- } \\
\text { tion) }\end{array}$} & & Traffic signal & No traffic signal & Total \\
\hline & $\begin{array}{l}\text { Traffic } \\
\text { signal }\end{array}$ & 42 & 3 & 45 \\
\hline & $\begin{array}{l}\text { No traffic } \\
\text { signal }\end{array}$ & 0 & 2 & 2 \\
\hline & Total & 42 & 5 & 47 \\
\hline
\end{tabular}

Table 2. The comparison of traffic signal state between proposed method and the real once

\begin{tabular}{|l|l|c|c|c|}
\hline & \multicolumn{4}{|c|}{ Real data (state) } \\
\hline \multirow{2}{*}{$\begin{array}{l}\text { The } \\
\text { pro- } \\
\text { posed } \\
\text { me- } \\
\text { thod } \\
\text { (state) }\end{array}$} & Green-Red & Green-Red & Amber Blinker & Total \\
\cline { 2 - 5 } & Total & 31 & 2 & 33 \\
\cline { 2 - 5 } & Amber Blinker & 3 & 11 & 14 \\
\hline
\end{tabular}

obtained from the experience of traffic engineers. The result of proposed method widely depends on these two parameters. In the conclusion, some methods are suggested for defining the better threshold to obtain more accurate result.

\section{Conclusions}

In this paper, an intelligent method was proposed to specify the spatial and non-spatial information of traffic signals. In reality, some conditions of the road such as destiny define the locations and states of traffic signals. As a result, in the geo-database, all spatial and non-spatial information of traffic signals are extracted from roads data while there is no need for defining and adding separate data for traffic signals into the database. The proposed method was the first step of a novel idea, so this method can be improved. Accurate estimation of $T^{\prime}, T_{1}$ and $T_{2}$ parameters will increase the efficiency of the proposed method. In this paper, $T_{2}$ could be obtained automatically; however, $T^{\prime}$ and $T_{1}$ are two parameters which must be defined in the algorithm manually. To overcome the weakness of the proposed method, a new approach is suggested. In the recommended method, these two parameters are considered as two unknown variants while the types and states of traffic signals are known. So these two unknown variants will be obtained by using mathematical optimization method. However, in this case, the information of traffic signals for some part of the network is needed. To overcome the weakness, it is also suggested that these two parameters for all traffic signals are considered as unknown. The unknown parameters, all AADTs and information of traffic signals of all roads are defined in a function. Therefore, the best values of these two parameters will be obtained by using optimization algorithm such as Genetic algorithm. Then, these two parameters are used as known constant parameters for the other studies.

\section{References}

'Awad, W. (2004). Estimating traffic capacity for weaving segments using neural networks technique. Applied Soft Computing Journal, 4(4), 395-404.

https://doi.org/10.1016/j.asoc.2004.01.006

Al Mufti, M., Al Hadhrami, E., Taha, B., \& Werghi, N. (2018). SAR automatic target recognition using transfer learning approach. In 2018 International Conference on Intelligent Autonomous Systems (ICoIAS), Singapore. IEEE. https://doi.org/10.1109/ICoIAS.2018.8494149

Annunziato, M., Bertini, I., Pannicelli, A., \& Pizzuti, S. (2003). Evolutionary feed-forward neural networks for traffic prediction. In Proc. of EUROGEN2003, Barcelona, Spain.

Behzadi, S., \& Alesheikh, A. A. (2014). Cellular automata vs. object-automata in traffic simulation. International Journal of Remote Sensing Applications, 4(1), 61-69.

Clay, M., \& Johnston, R. (2006). Multivariate uncertainty analysis of an integrated land use and transportation model: MEPLAN. Transportation Research Part D, 11(3), 191-203. https://doi.org/10.1016/j.trd.2006.02.001 
Fraile, A., Larrodé, E., Magreñán, Á. A., \& Sicilia, J. A. (2016). Decision model for siting transport and logistic facilities in urban environments: A methodological approach. Journal of Computational and Applied Mathematics, 291, 478-487. https://doi.org/10.1016/j.cam.2014.12.012

Fu, M., Kelly, J. A., \& Clinch, J. P. (2017). Estimating annual average daily traffic and transport emissions for a national road network: A bottom-up methodology for both nationally-aggregated and spatially-disaggregated results. Journal of Transport Geography, 58, 186-195.

https://doi.org/10.1016/j.jtrangeo.2016.12.002

Jabbari, M., \& Behzadi, S. (2019). Modelling effects of land use changes on traffic based on proposed traffic simulator. Computational Engineering and Physical Modeling, 2(3), 61-70. https://doi.org/10.22115/cepm.2020.207903.1073

Li, D., Cova, T. J., \& Dennison, P. E. (2019). Setting wildfire evacuation triggers by coupling fire and traffic simulation models: a spatiotemporal GIS approach. Fire Technology, 55(2), 617-642. https://doi.org/10.1007/s10694-018-0771-6

Mao, Y., Wang, Y., \& Zhang, L. (2008). Research on method of subsection learning of double- layers BP neural network in prediction of traffic volume. Jisuanji Gongcheng yu Yingyong (Computer Engineering and Applications), 44(13), 203-205.

Maoh, H., Kanaroglou, P., Scott, D., Paez, A., \& Newbold, B. (2009). IMPACT: An integrated GIS-based model for simulating the consequences of demographic changes and population ageing on transportation. Computers, Environment and Urban Systems, 33(3), 200-210.

https://doi.org/10.1016/j.compenvurbsys.2008.10.004
Maria, C., \& Gleriani, J. (2005). Cellular automata and neural networks as a modeling framework for the simulation of urban land use change. In Anais XII Simpósio Brasileiro de Sensoriamento Remoto (pp. 3697-3705).

Ocalir-Akunal, E. V. (2016). Decision support systems in transport planning. Procedia Engineering, 161, 1119-1126. https://doi.org/10.1016/j.proeng.2016.08.518

Ozkurt, C., Camci, F., \& Stanbul, T. (2009). Automatic traffic density estimation and vehicle classification for traffic surveillance systems using neural networks. Mathematical and Computational Applications, 14(3), 187-196. https://doi.org/10.3390/mca14030187

Shaw, S., \& Xin, X. (2003). Integrated land use and transportation interaction: a temporal GIS exploratory data analysis approach. Journal of Transport Geography, 11(2), 103-115. https://doi.org/10.1016/S0966-6923(02)00070-4

Srinivasan, D., Choy, M., \& Cheu, R. (2006). Neural networks for real-time traffic signal control. IEEE Transactions on Intelligent Transportation Systems, 7(3), 261-272. https://doi.org/10.1109/TITS.2006.874716

Wang, X. (2005). Integrating GIS, simulation models, and visualization in traffic impact analysis. Computers, Environment and Urban Systems, 29(4), 471-496.

https://doi.org/10.1016/j.compenvurbsys.2004.01.002

Ying, J. (2007). Continuous optimization method for integrated land use/transportation models. Journal of Transportation Systems Engineering and Information Technology, 7(3), 64-72. https://doi.org/10.1016/S1570-6672(07)60022-1 